Meeting report

\title{
American Society for Clinical Oncology 39th Annual Meeting, Chicago, Illinois, USA, 31 May to 3 June 2003: Breast cancer neoadjuvant and adjuvant chemotherapy - prognostic and predictive markers
}

\author{
Helena Earl \\ Correspondence: Helena Earl (e-mail: hme22@cam.ac.uk) \\ Published: 5 September 2003 \\ Breast Cancer Res 2003, 5:289-293 (DOI 10.1186/bcr647) \\ (C) 2003 BioMed Central Ltd (Print ISSN 1465-5411; Online ISSN 1465-542X)
}

Department of Oncology, Box 193, University of Cambridge, Addenbrookes Hospital, Cambridge, UK

\begin{abstract}
The annual American Society of Clinical Oncology congress is the largest forum for cancer professionals in the world, promoting new developments in cancer medicine. A wide spectrum of subjects relevant to breast cancer research and treatment was covered in this year's meeting, including chemotherapy in the neoadjuvant and adjuvant settings, molecular and gene profiling studies, and use of more conventional prognostic and predictive markers of outcome. This report discusses some of the highlights in translational and clinical aspects of early breast cancer management presented at the meeting.
\end{abstract}

Keywords: adjuvant, chemotherapy, gene profile, marker, neoadjuvant, predictive, prognostic

\section{Introduction}

The American Society of Clinical Oncology (ASCO) Annual Meeting is a leading educational and scientific event for oncologists, clinical researchers, academics and other health care professionals involved in multidisciplinary cancer care. This year the congress was held in Chicago, Illinois, from May 31 to June 3. The theme for the 39th annual meeting was 'Commitment, care, compassion: honouring people with cancer'. The goal of the meeting was to promote communication among cancer related medical specialities and the exchange of ideas arising from ongoing advances in oncology. This encompassed the areas of pathophysiology, diagnosis and management, and included innovations in therapies. For the first time this year, an oral presentation session was devoted to pharmacogenomics. A wide range of translational scientific research relevant to breast cancer was covered as well as new clinical data pertinent to breast oncology management.

\section{Gene profiling in translational breast cancer research}

Worldwide, many research groups are concentrating on breast cancer gene expression and molecular profiling, and this area was given significant coverage at the ASCO meeting. The first presentation in the meeting's plenary session, given by Lajos Pusztai from the MD Anderson group, dealt with the predictive nature of profiling in terms of response to chemotherapy. Their group described the use of gene expression profiling in predicting complete pathological response ( $p C R$ ) to neoadjuvant chemotherapy with a paclitaxel and anthracycline combination (abstract \#1 [1]). In 21 patients the overall accuracy of response prediction based on a group of five genes (three oestrogen sulphotransferases, nuclear factor 1/A, and histone acetyltransferase) was $81 \%$ and the positive predictive value for $\mathrm{PCR}$ was $75 \%$, with an overall specificity of $93 \%$, although sensitivity fell to $50 \%$. 
The group from Baylor College (abstract \#32 [1]) presented their work on gene expression patterns for de novo and acquired resistance to docetaxel. Twenty-four patients had paired samples before and after four cycles of docetaxel $100 \mathrm{mg} / \mathrm{m}^{2}$. Docetaxel resistance occurred in $54 \%$ of the tumours and $46 \%$ were sensitive. Resistant tumours showed elevated baseline expression of some transcriptional genes and genes involved in repair/synthesis. Docetaxel-sensitive tumours showed higher baseline expression of mitochondrial proteins related to apoptosis. After 3 months of docetaxel chemotherapy, both the resistant and the sensitive groups exhibited the 'resistant' profile, suggesting that de novo and acquired chemotherapy resistance have the same or similar genotype. This work has now been published in detail in the Lancet [2].

The group from Yamato, Japan (abstract \#34 [1]), looked at oligonucleotide microarrays in 20 patients receiving neoadjuvant chemotherapy with doxyfluoridine $800 \mathrm{mg} / \mathrm{m}^{2}$ days $1-14$ and docetaxel $60 \mathrm{mg} / \mathrm{m}^{2}$ day 8 every 3 weeks for four cycles. Out of 76 genes, among the good responders 15 genes were upregulated, including diubiquitin, ICAM-3, N-myc, and RNA-helicase, whereas 13 genes were downregulated.

Clearly, the hope is that molecular profiling in breast cancer will provide both a prognostic tool, as described elegantly by van 't Veer and coworkers [3] in their seminal paper published in Nature, and also a tool for predicting response to different chemotherapeutic agents. Molecular profiling may also identify groups of genes, which may provide targets - at a single gene and a pathway level for the development of more specific therapeutic molecules.

\section{Neoadjuvant chemotherapy}

The neoadjuvant setting in breast cancer provides excellent opportunities as a 'biomarker discovery laboratory' (abstract \#33 [1]) and a test ground for newer treatment combinations. A number of abstracts focused on primary systemic chemotherapy, including the molecular translational research discussed above. Efforts to identify good predictive factors for response are well placed because the outcome measure occurs early and can inform research on larger trial samples in the adjuvant setting.

The important features of the neoadjuvant presentations are summarized in Table 1. pCR is highlighted as the most significant early outcome measure in these studies. We understand from the work of Chollet and coworkers [4] that, irrespective of initial prognostic factors in a tumour, when $\mathrm{pCR}$ is achieved this correlates with a disease-free survival of over $80 \%$ at 10 years. The highest $\mathrm{pCR}$ seen in this group of abstracts is that from Limentani (abstract \#131 [1]), with a $36 \%$ pCR to docetaxel with granulocyte colony-stimulating factor (GCSF) support, with the addition of herceptin in the HER2-positive population.

Perhaps the most noteworthy of the neoadjuvant abstracts is that from Ramirez-Ugalde and coworkers from Mexico City (abstract \#160 [1]). Patients with stable or progressive disease following standard anthracycline-based neoadjuvant chemotherapy were treated with weekly 5-fluorouracil, gemcitabine and dexamethasone during radiotherapy to the breast ( 50 Gy with 10 Gy boost). A pCR rate of $28 \%$ was noted, with attainment of minimal residual disease in a further $23 \%$, making surgery possible in 47 out of 49 patients. This is a truly remarkable achievement in this group of breast cancer patients selected for their refractoriness to anthracyclines. Further evaluation of the combination of chemotherapy and radiotherapy may be useful in this poor prognosis group.

\section{Early breast cancer: adjuvant chemotherapy}

Mamounas presented the updated results of the NSABPB28 study, which examined the addition of four cycles of paclitaxel $225 \mathrm{mg} / \mathrm{m}^{2}$ to the standard of four cycles of doxorubicin and cyclophosphamide (abstract \#12 [1]). A total of 3060 patients were randomized, and the study now has a median follow up of 64 months. Disease-free survival shows a relative risk for relapse of 0.83 $(P=0.008)$ in favour of the addition of paclitaxel, although overall survival did not show the same advantage (relative risk 0.94, $P=0.46$ ). There appeared to be no interaction of taxanes with oestrogen receptor (ER) positivity, although a confounding factor may be the concomitant treatment of ER-positive patients with tamoxifen. There are at present no significant differences in death rates between the standard and experimental arm. This is perhaps because the prognosis of patients in the study is very good and that at median follow up of 64 months it is too early to detect any differences. This study supports the earlier findings of CALGB 9344 [5], which indicated benefit from the addition of taxanes, although the CALGB 9344 study suggested a definite interaction between ER status and benefit from taxanes. In ER-negative disease there are now more studies showing lack of interaction than studies showing positive interaction [6].

This report reiterates the difference in biology between hormone receptor (HR) positive and negative disease. Analysis of relapse and survival in the first 5 years is more informative for the HR-negative group, in which the majority of events occur within this time frame, rather than for the HR-positive population, in which events occur more often after 5 years (abstract \#55 [1]). Similarly, the interaction of chemotherapy treatments appears to produce more effect in the HR-negative population within the first 5 years, and in the HR-positive population after this time [7]. This must be remembered when analyzing adjuvant 
Table 1

\begin{tabular}{|c|c|c|c|}
\hline Abstract no. & Treatment & $n$ & $\mathrm{pCR}$ \\
\hline 1 & Paclitaxel weekly and FAC & 24 & $\begin{array}{l}25 \% \\
\text { (breast and lymph nodes) }\end{array}$ \\
\hline 32 & Docetaxel $100 \mathrm{mg} / \mathrm{m}^{2} \mathrm{q} 3$ weeks $\times 4$ & 24 & $0 \%$ \\
\hline 33 & Doxorubicin $75 \mathrm{mg} / \mathrm{m}^{2} \times 3$; then docetaxel $40 \mathrm{mg} / \mathrm{m}^{2} \mathrm{q}$ week $\times 6$ & 70 & $12.5 \%$ \\
\hline 34 & Doxyfluoridine $800 \mathrm{mg} / \mathrm{m}^{2} \mathrm{D} 1-14$; docetaxel $60 \mathrm{mg} / \mathrm{m}^{2} \mathrm{D} 8 \mathrm{q} 3$ weeks & 20 & $14 \%$ \\
\hline 35 & Paclitaxel doxorubicin standard or DD $\times 6$ & 460 & $\begin{array}{l}\mathrm{ER}^{-} 26 \% \\
\mathrm{ER}^{+} 8 \%\end{array}$ \\
\hline 37 & Paclitaxel + doxorubicin $\times 4$ then CMF $\times 4$ & 451 & $23 \%$ \\
\hline 80 & Epirubicin $120 \mathrm{mg} / \mathrm{m}^{2} \mathrm{q} 3$ weeks \pm tamoxifen & 211 & $\begin{array}{l}\mathrm{ER}^{-} 23.4 \% \\
\mathrm{ER}^{+} 4.6 \%\end{array}$ \\
\hline 83 & Vinorelbine $25 \mathrm{mg} / \mathrm{m}^{2} \mathrm{D} 1+8$ and epirubicin $60 \mathrm{mg} / \mathrm{m}^{2} \mathrm{D} 1$ versus $\mathrm{AC} \times 6$ & 411 & $15 \%$ both arms \\
\hline 85 & $\begin{array}{l}\text { TAC } \times 2: \text { then PR to TAC } \times 6(107 \text { patients }) \\
<\mathrm{PR} \text { to TAC } \times 4(24 \text { patients }) \\
\text { or vinorelbine and xeloda } \times 4(20 \text { patients })\end{array}$ & 151 & $\begin{array}{l}19 \% \\
26 \% \text { for clinical } \\
\text { responders to TAC }\end{array}$ \\
\hline 86 & Vinorelbine/herceptin & 28 & $\begin{array}{l}29 \% \\
\text { cCR+PR } 93 \%\end{array}$ \\
\hline 107 & Epirubicin $60 \mathrm{mg} / \mathrm{m}^{2} \mathrm{D} 1$, CDDP $60 \mathrm{mg} / \mathrm{m}^{2} \mathrm{D} 1$, xeloda $1000 \mathrm{mg} / \mathrm{m}^{2}$ BD D1-14 & 48 & $27 \%$ \\
\hline 131 & Docetaxel $60 \mathrm{mg} / \mathrm{m}^{2}+$ vinorelbine $45 \mathrm{mg} / \mathrm{m}^{2} \mathrm{q} 2$ weeks + GCSF + herceptin in HER2 ${ }^{+}$ & 33 & $36 \%$ \\
\hline 140 & Gemcitabine $1 \mathrm{~g} / \mathrm{m}^{2}$, epirubicin $90 \mathrm{mg} / \mathrm{m}^{2}$, paclitaxel $175 \mathrm{mg} / \mathrm{m}^{2} \mathrm{q} 3$ weeks & 22 & $22.7 \%$ \\
\hline 143 & Docetaxel $100 \mathrm{mg} / \mathrm{m}^{2} \mathrm{q} 3$ weeks $\times 4$ & 42 & $10 \%$ \\
\hline 160 & $\begin{array}{l}\text { Anthracycline SD or PD; } 5 \text { FU } 500 \mathrm{mg} / \mathrm{m}^{2} / \mathrm{w}+\text { gemcitabine } 100 \mathrm{mg} / \mathrm{m}^{2} / \mathrm{w} \\
+ \text { dexamethasone } 16 \mathrm{mg} \text { throughout radiotherapy }(50 \mathrm{~Gy}+10 \mathrm{~Gy} \text { boost })\end{array}$ & 47 & $\begin{array}{l}28 \% \\
\text { MRD (+pNo) } 23 \%\end{array}$ \\
\hline 163 & $\begin{array}{l}\text { Docetaxel } 75 \mathrm{mg} / \mathrm{m}^{2}+\text { doxorubicin } 50 \mathrm{mg} / \mathrm{m}^{2}+\text { cyclophosphamide } \\
500 \mathrm{mg} / \mathrm{m}^{2} \times 4 \mathrm{q} 3 \text { weeks }\end{array}$ & 37 & $8 \%$ \\
\hline 190 & Docetaxel $100 \mathrm{mg} / \mathrm{m}^{2} \mathrm{q} 3$ weeks $\times 6$ & 88 & $19.8 \%$ \\
\hline
\end{tabular}

CMF, cyclophosphamide, methotrexate and fluorouracil; ER, oestrogen receptor; 5FU, 5-fluorouracil; FAC, 5-fluorouracil, doxorubicin and cyclophosphamide; GCSF, granulocyte colony-stimulating factor; pCR, complete pathological response; TAC, docetaxel, doxorubicin and cyclophosphamide; PR, partial response; $\angle \mathrm{PR}$, less than a partial response; cCR, complete clinical response; CDDP, cisplatin; DD, dose dense; $\mathrm{SD}$, stable disease; PD, progessive disease; MRD, minimal residual disease; $+\mathrm{pNO}$, pathological node negative.

studies that include a high proportion of HR-positive women $(66 \%$ in this study).

The National Epirubicin Adjuvant Trial (NEAT) trial (abstract \#13 [1]) was presented by Dr Chris Poole from the Cancer Research UK Institute and Cancer Trials Unit in Birmingham. This is the first of the large adjuvant chemotherapy trials to come from the UK, and shows a very significant advantage for epirubicin, cyclophosphamide, methotrexate and fluorouracil (ECMF) chemotherapy in terms of both disease-free and overall survival when compared with standard classical cyclophosphamide, methotrexate and fluorouracil (CMF) for six cycles. The study is the most strongly positive of the very few individual trials that demonstrate an advantage for anthracycline-based chemotherapy. This advantage is demonstrated across all stratification groups. The study has been reported as a preplanned meta-analysis with a smaller trial run by the Scottish Cancer Therapy Network, which asked broadly similar questions. The event-driven analysis was carried out 18 months later than anticipated, suggesting that the results for patients treated on the standard CMF arm were considerably better than had been predicted before the trial started.

The trial has both proved an advantage for the Bonadonna block-scheduling anthracycline approach [8], and improved outcomes for breast cancer patients in the UK by insisting on adherence to the two classical CMF schedules (oral or intravenous cyclophosphamide). Direct comparisons in the metastatic setting had already shown three-weekly CMF to be inferior, and the analysis by Bonadonna and coworkers of their own original CMF study [9] showed that less than $85 \%$ dose delivery of the 
classical CMF resulted in inferior outcomes. At ASCO 2002 the group reported that the ECMF regimen is tolerable [10]. This study provided further confirmation of the benefit from anthracycline-based treatment and shows that, unlike four cycles of doxorubicin and cyclophosphamide, which was shown to be equivalent to CMF, the ECMF block-scheduling is definitely superior. The emergence of acute myeloid leukaemia/myelodysplastic syndrome in the fluorouracil, epirubicin and cyclophosphamide (FEC) D1 and 8 studies (the only other studies to show in a 'stand alone way' the superiority of anthracyclines) raises concerns [11]. To date no cases of acute myeloid leukaemia have been reported in the NEAT and Scottish studies, although at present follow up is shorter.

Following the recently successful CALGB 9471 adjuvant study [12], which showed a strongly positive result for dose dense therapy, it is of considerable interest to see early reports of piloted adjuvant treatments from this group. Abstract 46 [1] described an adjuvant phase II study of dose dense FEC followed by weekly paclitaxel and docetaxel. This pilot study looked at both feasibility and efficacy. FEC $\left(500 / 100 / 500 \mathrm{mg} / \mathrm{m}^{2}\right)$ was administered every 2 weeks for six cycles with GCSF support, followed by weekly paclitaxel $\left(80 \mathrm{mg} / \mathrm{m}^{2}\right)$ and docetaxel $\left(35 \mathrm{mg} / \mathrm{m}^{2}\right)$ for 18 weeks (a total of 30 weeks of treatment). This schedule produced significant toxicity with grade $3 / 4$ pneumonitis in four out of $44(9 \%)$, requiring prolonged hospitalization. This complication seemed wholly attributable to the dose dense FEC. Seventeen patients proceeded with the second phase of weekly taxanes for 18 weeks, and after completion of this two out of 17 patients (11\%) developed severe pleural and pericardial effusions, again necessitating hospitalization. This protocol is not being further developed for high-risk patients, and may interestingly set a new limit to the amount of intensification achievable with GCSF support alone rather than stem cell haematological support.

Fumoleau (abstract \#91 [1]) presented data for the French Adjuvant Study Group (FASG). This was a 7-year analysis of the benefit/risk ratio of epirubicin in adjuvant chemotherapy trials (3577 patients). When used at classical doses up to epirubicin $100 \mathrm{mg} / \mathrm{m}^{2}$ per cycle, the benefit/risk ratio in operable breast cancer remains in favour of epirubicin-based adjuvant chemotherapy. A 10-year update of the FASG 05 trial (FEC50 versus FEC100) presented by Bonneterre (abstract \#93 [1]), analyzing benefit/risk ratio after adjuvant chemotherapy in node-positive early breast cancer patients, confirmed the advantage at 10 years of FEC 100 over FEC50.

\section{Prognostic/predictive markers (conventional)}

A number of abstracts were presented on more conventional tumour markers as both predictive and prognostic
(BCTMAP; abstract \#9 [1]) examined the impact of cyclooxygenase (COX)-2, HER2 and aromatase expression in 930 patients with breast cancer enrolled in phase II/III clinical trials. Expression of HER2 and COX-2 but not aromatase was associated with poorer breast cancer specific survival; however, positivity for both COX-2 in addition to HER2 did not increase the relative risk for death related to breast cancer when compared with all other groups. The data were felt to support the likelihood of interactions between COX-2, HER2 and aromatase pathways.

The Austrian Breast and Colorectal Cancer Study Group (ABCSG) reported on 512 patients in ABCSG Trial 5 (abstract \#10 [1]). Premenopausal HR-positive patients were randomized between CMF chemotherapy and combined hormonal therapy with tamoxifen and goserelin. High p27Kip1 expression was observed in 413 patients. Combination endocrine therapy was superior to CMF in patients with high p27Kip1 expression but not in low expressing patients. Adjusted relative risks for relapse and death after combined endocrine therapy when compared with CMF were as follows: high p27Kip1 0.52 (95\% confidence interval $0.32-0.83 ; P=0.006$ ) and 0.51 (95\% confidence interval $0.21-1.25 ; P=0.14$ ) for relapse and death, respectively. In women with low p27Kip1 expression there was no difference in response for CMF or combined hormone therapy. These results suggest that p27Kip1 may be a useful marker for selection of patients for combined endocrine therapy in this premenopausal group. ER status was shown in neoadjuvant trials to predict response to neoadjuvant chemotherapy (abstracts \#35 and \#80 [1]). ER-negative patients had higher rates of $\mathrm{pCR}$ to chemotherapy. Increased responsiveness is also related to Ki67 positivity. This is confirmatory evidence to support the hypothesis of interaction between different tumour biology (ER-positive versus ER-negative disease) and response to chemotherapy. Recent clinical data from trials sequencing tamoxifen and chemotherapy support the use of sequential hormone therapy after completion of chemotherapy to produce better outcomes for patients.

Ravdin and coworkers (abstract \#55 [1]) looked at the predictive factor of ER-negative tumours in the adjuvant setting. They concluded that ER-negative status should not be used as the sole criteria for deciding on adjuvant chemotherapy in node-negative patients. This poster presented some interesting survival curves from diagnosis comparing the ER-negative group with the ER-positive one. The prevalence of death from breast cancer rose quickly in the ER-negative group and peaked at 3 years with only a small dip at 4 years, before a 'twin peak' at 5 years. The ER-positive curve showed a slow increase in risk for recurrence through time with the two survival curves crossing at 8.5 years from diagnosis. These survival curves are of considerable interest and attest to the 
different tumour biology of ER-positive and ER-negative disease, and to the way in which these two different types of breast cancer might interact with chemotherapy treatment.

The group from the MD Anderson Cancer Center (abstract \#56 [1]) reported on the prognostic value of epidermal growth factor receptor expression, and its ability to add to the prognostic effect of HER2 over-expression. The Guy's Group (abstract \#144 [1]) with the MRC Cancer Trials Unit presented their estimates for overall survival probabilities and probable benefit for adjuvant systemic therapy (both hormonal and chemotherapy). The predicted survival at 10 years from this model of a 55-year-old with a $30 \mathrm{~mm}$ grade 3 tumour with two positive nodes was as follows: no treatment 38\%; chemotherapy alone 47\%; hormonal therapy for ER-positive tumour 64\%; and hormonal therapy plus chemotherapy 69\%. This represents an improvement in absolute terms of 31\% in 10-year survival, with substantial contributions from both chemotherapy and hormonal therapy.

\section{Conclusion}

The 39th ASCO Annual Meeting showcased many interesting developments in clinical and translational research that are relevant to breast cancer, allowing dissemination of useful information to people working in cancer related medical specialities. Improved understanding of the pathogenesis of breast cancer, emerging prognostic and predictive strategies, and developments in clinical data for therapeutic interventions will significantly influence our breast cancer practice in the future.

\section{Competing interests}

$\mathrm{HE}$ received an educational grant from Eli Lilly Co Ltd (UK) to attend the 2003 ASCO meeting.

\section{References}

1. Proceedings of the American Society of Clinical Oncology. 39th Annual Meeting, Chicago, Illinois. J Clin Oncol 2003, 21.

2. Chang JC, Wooten EC, Tsimelzou A, Hilsenbeck SG, Gutierrez MC, Elledge R, Mohsin S, Osborne CK, Chamness GC, Allred DC, and O'Connell P: Gene expression profiling for the prediction of therapeutic response to docetaxel in patients with breast cancer. Lancet 2003; 363: 362-369.

3. van 't Veer LJ, Dai $H$, van de Vijver MJ, He YD, Hart AA, Mao M, Peterse HL, van der Kooy K, Marton MJ, Witteveen AT, Schreiber GJ, Kerkhoven RM, Roberts C, Linsley PS, Bernards R, Friend $\mathrm{SH}$ : Gene expression profiling predicts clinical outcome of breast cancer. Nature 2002, 415:530-536.

4. Chollet P, Amat S, Cure H, de Latour M, Le Bouedec G, MouretReynier MA, Ferriere JP, Achard JL, Dauplat J, Penault-Llorca F: Prognostic significance of a complete pathological response after induction chemotherapy in operable breast cancer. $\mathrm{Br} J$ Cancer 2002, 86:1041-1046.

5. Henderson IC, Berry DA, Demetri GD, Cirrincione CT, Goldstein LJ, Martino S, Ingle JN, Cooper MR, Hayes DF, Tkaczuk KH, Fleming G, Holland JF, Duggan DB, Carpenter JT, Frei E 3rd, Schilsky RL, Wood WC, Muss HB, Norton L: Improved outcomes from adding sequential paclitaxel but not from escalating doxorubicin dose in an adjuvant chemotherapy regimen for patients with node-positive primary breast cancer. J Clin Oncol 2003, 21:976-983.
6. Nabholtz JM, Pienkowski T, Mackey J, Pawlicki M, Guastella JP, Vogel C, Weaver C, Walley B, Martin M, Chap L, Tomiak E, Juhos E, Guevin R, Howell A, Hainsworth J, Fornander T, Blitz S, Gazel S, Loret C, Riva A: Phase III trial comparing TAC (docetaxel, doxorubicin, cyclophosphamide) in the adjuvant treatment of node positive breast cancer (BC) patients: interim analysis of the BCIRG 001 study. Proc ASCO [abstract]. J Clin Oncol 2002, 21:141.

7. Valero V, Buzdar AU, McNeese M, Singletary E, Hortobagyi GN: Primary chemotherapy in the treatment of breast cancer: the University of Texas M. D. Anderson Cancer Center experience. Clin Breast Cancer 2002, suppl 2:S63-S68.

8. Bonadonna G, Zambetti M, Valagussa P: Sequential or alternating doxorubicin and CMF regimens in breast cancer with more than three positive nodes. JAMA 1995, 273:542-547.

9. Bonadonna G, Valagussa P, Moliterni A, Zambetti M, Brambilla C: Adjuvant cyclophosphamide, methotrexate, and fluorouracil in node-positive breast cancer, the results of 20 years of followup. N Engl J Med 1995, 332:901-906.

10. Earl HM, Poole CJ, Dunn J, et al., on behalf of the NEAT Steering Committee: NEAT - National Epirubicin Adjuvant Trial. A multicentre phase III randomised trial of epirubicin $\times 4$ and classical CMF $\times 4$ (ECMF) versus CMF $\times 6$ (CMF). Proc ASCO [abstract]. J Clin Oncol 2002, 21:2050.

11. Levine MN, Bramwell VH, Pritchard KI, Norris BD, Shepherd LE Abu-Zahra H, Findlay B, Warr D, Bowman D, Myles J, Arnold A, Vandenberg T, MacKenzie R, Robert J, Ottaway J, Burnell M, Williams CK, Tu D: Randomised trial of intensive cyclophosphamide, epirubicin, and fluorouracil chemotherapy compared with cyclophosphamide, methotrexate, and fluorouracil in premenopausal women with node-positive breast cancer. $J$ Clin Oncol 1998, 16:2651-2658.

12. Citron ML, Berry DA, Cirrincione C, Hudis C, Winer EP, Gradishar WJ, Davidson NE, Martino S, Livingston R, Ingle JN, Perez EA, Carpenter J, Hurd D, Holland JF, Smith BL, Sartor Cl, Leung EH, Abrams J, Schilsky RL, Muss HB, Norton L: Randomized trial of dose-dense versus conventionally scheduled and sequential versus concurrent combination chemotherapy as postoperative adjuvant treatment of node-positive primary breast cancer: first report of Intergroup Trial C9741/Cancer and Leukemia Group B Trial 9741. J Clin Oncol 2003, 21:14311439.

\section{Correspondence}

Helena Earl, University of Cambridge, Department of Oncology, Addenbrookes Hospital (Box 193), Cambridge, CB2 2QQ, UK. Tel: +44 01223336 800; fax: +44 01223763 120; e-mail: hme22@cam.ac.uk 